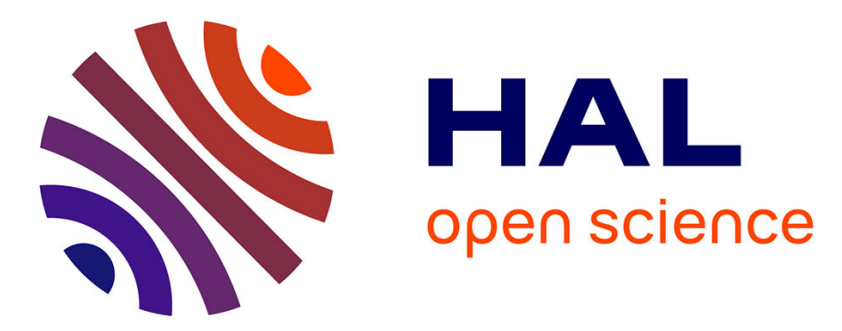

\title{
Influence de la température sur la vitesse limite des trous dans un transistor MOS à canal court de type $\mathbf{P}$
}

M. Gamboa, T. Phan Pham, Henri Tranduc, P. Rossel

\section{To cite this version:}

M. Gamboa, T. Phan Pham, Henri Tranduc, P. Rossel. Influence de la température sur la vitesse limite des trous dans un transistor MOS à canal court de type P. Revue de Physique Appliquée, 1981, 16 (10), pp.597-599. 10.1051/rphysap:019810016010059700 . jpa-00244952

\section{HAL Id: jpa-00244952 https://hal.science/jpa-00244952}

Submitted on 1 Jan 1981

HAL is a multi-disciplinary open access archive for the deposit and dissemination of scientific research documents, whether they are published or not. The documents may come from teaching and research institutions in France or abroad, or from public or private research centers.
L'archive ouverte pluridisciplinaire HAL, est destinée au dépôt et à la diffusion de documents scientifiques de niveau recherche, publiés ou non, émanant des établissements d'enseignement et de recherche français ou étrangers, des laboratoires publics ou privés. 


\title{
Influence de la température sur la vitesse limite des trous dans un transistor MOS à canal court de type $\mathbf{P}$
}

\author{
M. Gamboa, T. Phan Pham, H. Tranduc et P. Rossel \\ Laboratoire d'Automatique et d'Analyse des Systèmes du C.N.R.S., \\ 7, avenue du Colonel-Roche, 31400 Toulouse, France
}

(Reçu le 15 avril 1981, révisé le 8 juillet 1981, accepté le 15 juillet 1981)

\begin{abstract}
Résumé. - On détermine pour la première fois, la variation de la vitesse de saturation des trous en fonction de la température dans un transistor MOS, à canal $\mathrm{P}$, dont la surface est orientée (111). Il est montré que cette variation est du type exponentiel, ce qui semblerait être un mode d'évolution général indépendant de la nature des porteurs ou de l'orientation cristallographique.
\end{abstract}

Abstract. - The temperature dependence of the saturation velocity in P short channel MOS transistor (111) is determined.

It turns out that the dependence is exponential. It appears that this result is a general kind of evolution, independently of the carriers nature or the crystal orientation.

1. Introduction. - Dans les transistors MOS des nouvelles générations, à canal court, les porteurs transitent à leur vitesse limite, entre la source et le drain. Cette vitesse limite est un facteur de premier ordre qui détermine les valeurs des paramètres électriques du composant [1]. A ce jour, il n'existe que peu de renseignements $[2,3]$ sur les valeurs ou les propriétés théoriques de cette vitesse, particulièrement en ce qui concerne les trous. Dans cette communication, nous nous proposons de déterminer expérimentalement les valeurs et le mode d'évolution en température de la vitesse limite de ces trous dans une couche inversée de silicium orienté (111). Le principe utilisé est le même que celui que nous avions mis en œuvre précédemment dans le cas des électrons [4].

2. Principe de la méthode. - D'un point de vue théorique, en utilisant d'une part l'expression de la partie imaginaire de l'admittance de sortie $Y(\omega)$ du transistor MOS à canal court [5] :

$$
\operatorname{Im}[Y(\omega)]=\operatorname{Im}\left[Z_{\mathrm{th}}(\omega)\right] \cdot I_{\mathrm{D}} \cdot \frac{\partial I_{\mathrm{D}}}{\partial T}
$$

et d'autre part la relation approchée qui définit le courant drain $[1,6]$

$$
I_{\mathrm{D}}=Z \cdot C_{O X} \cdot v_{\mathrm{sat}} \cdot\left(V_{\mathrm{G}}-V_{\mathrm{T}}\right) .
$$

On obtient l'équation différentielle à laquelle satisfait la vitesse limite $v_{\text {sat }}[4]$ :

$$
\frac{\partial \log v_{\text {sat }}}{\partial T}=\frac{\operatorname{Im}[Y(\omega)]_{\max }}{\operatorname{Im}\left[Z_{\mathrm{th}}(\omega)\right]_{\max } \cdot I_{\mathrm{D}} \cdot\left(I_{\mathrm{D}}-I_{\mathrm{C}}\right)} .
$$

Dans ces relations, $I_{\mathrm{D}}$ est le courant de drain, $T$ la température de cristal de silicium, $Z_{\mathrm{th}}(\omega)$ l'impédance thermique de l'ensemble "puce-boîtier " à la pulsation $\omega, Z . C_{O X}$ la capacité de grille par unité de longueur du canal, $V_{\mathrm{T}}$ la tension du seuil du transistor, $\operatorname{Im}[Y(\omega)]_{\max }$ la valeur maximale de la partie imaginaire de l'admittance de sortie et $I_{C}$ la valeur du courant qui correspond à l'annulation de cette valeur $\operatorname{Im}[Y(\omega)]_{\max }$. La technique proposée pour obtenir la loi d'évolution $v_{\text {sat }}(T)$ consiste à déterminer expérimentalement les différents termes du deuxième membre de la relation (3) puis d'intégrer cette relation.

3. Résultats expérimentaux. - Les transistors étudiés sont des structures V MOS interdigitées dont le canal de type $\mathrm{P}$ a une longueur inférieure à $2 \mu \mathrm{m}$. La longueur $Z$ de grille est de $7 \mathrm{~cm}$ et l'épaisseur d'oxyde est égale à $1000 \AA$. La surface du canal est orientée (111).

Pour ces transistors, on mesure le diagramme d'admittance électrique de sortie $Y(\omega)$ à l'aide du banc de mesure décrit dans la référence [5] ; une forme typique est représentée sur la figure 1. Le diagramme 


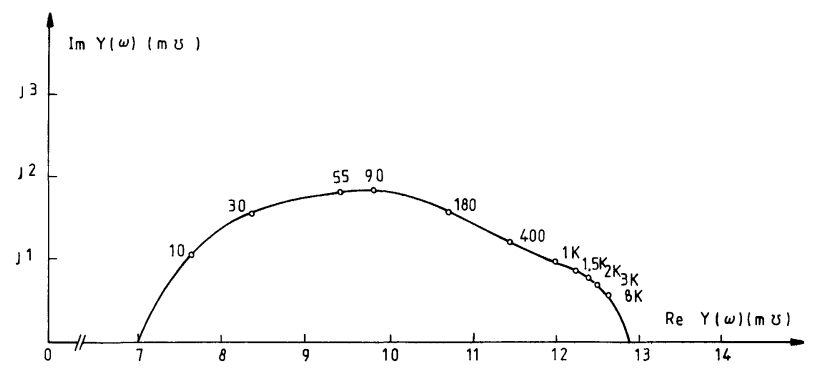

Fig. 1. - Diagramme d'admittance typique obtenu expérimentalement. Le paramètre est la fréquence en $\mathrm{Hz}$ ou $\mathrm{kHz} .\left(V_{\mathrm{D}}=15 \mathrm{~V}\right.$, $\left.I_{\mathrm{D}}=1 \mathrm{~A}, T_{\text {boitier }}=300 \mathrm{~K}\right)$.

[Typical diagram of admittance. The parameter is the frequency.]

d'admittance dépend du point de fonctionnement $\left(V_{\mathrm{D}}, I_{\mathrm{D}}, T\right)$. Nous obtenons ainsi :

(i) Les variations de la valeur maximale İm $[Y(\omega)]_{\max }$ de la partie imaginaire de $Y(\omega)$. Celle-ci est une fonction parabolique du courant de drain [5]. Elle s'annule pour la valeur particulière $I_{\mathrm{C}}$. Les variations expérimentales de cette quantité Im $[Y(\omega)]_{\max }$ en fonction de $I_{\mathrm{D}}\left(I_{\mathrm{D}}-I_{\mathrm{C}}\right)$ sont linéaires comme le montre la figure 2. La pente de la droite obtenue est indépendante, à $10 \%$ près de la température dans la gamme de température de cristal $77 \mathrm{~K}-400 \mathrm{~K}$. En conséquence, on peut écrire que ces résultats expérimentaux se traduisent par :

$$
\operatorname{Im}[Y(\omega)]_{\max }=\alpha \cdot I_{\mathrm{D}} \cdot\left(I_{\mathrm{D}}-I_{\mathrm{C}}\right) .
$$

où la constante $\alpha$ est égale à : $1,9 \times 10^{-3} \mho \cdot \mathrm{A}^{-2}$.

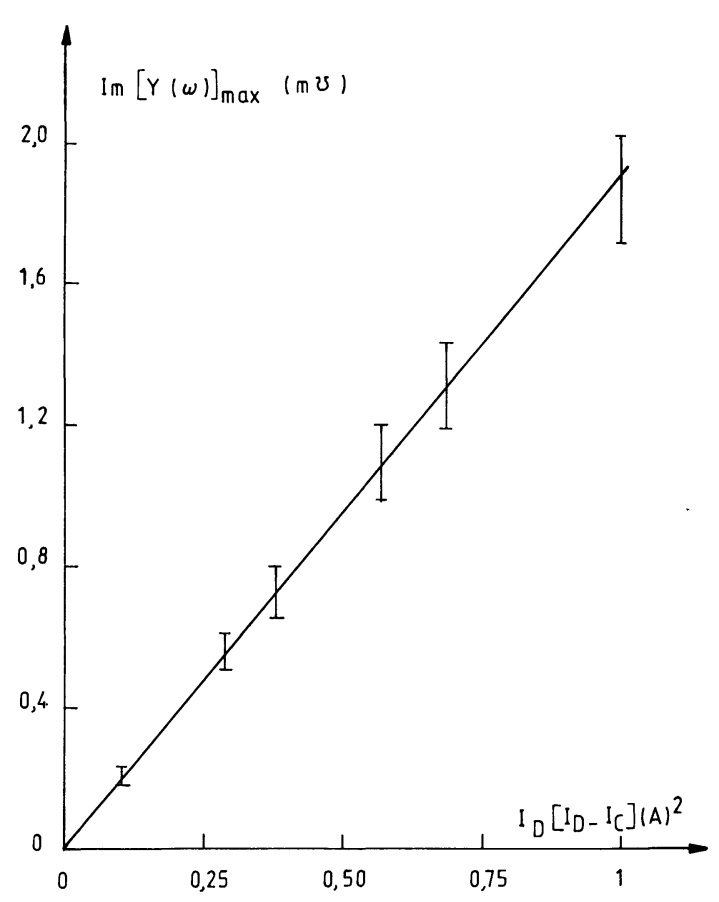

Fig. 2. - Variations expérimentales de $\operatorname{Im}[Y(\omega)]_{\max }$ en fonction du produit $I_{\mathrm{D}}\left(I_{\mathrm{D}}-I_{\mathrm{C}}\right)$, dans la gamme de température du boîtier comprise entre $77 \mathrm{~K}<T<400 \mathrm{~K}$. $\Phi$ expérience; — relation (4)

[Experimental values of $\operatorname{Im}[Y(\omega)]_{\max }$ versus $\left.I_{\mathrm{D}}\left(I_{\mathrm{D}}-I_{\mathrm{C}}\right) \cdot\right]$ (ii) La partie imaginaire de l'impédance thermique est obtenue par la méthode décrite dans la référence [5]. Elle est égale à $1\left(^{\circ}\right) \mathrm{W}^{-1}$ à $350 \mathrm{~K}$ de température de cristal et peut être considérée comme indépendante de la température dans la gamme $200 \mathrm{~K}-400 \mathrm{~K}$.

4. La vitesse limite. - Par intégration de la relation (3), en tenant compte de (4), il vient :

$$
v_{\text {sat }}=v_{\text {sat } 0} \cdot \exp \left[-\beta \cdot\left(T-T_{0}\right)\right] .
$$

$v_{\text {sat } 0}$ est la vitesse de saturation à la température $T_{0}$, sa valeur numérique est déduite d'une mesure de transconductance en régime saturé et de la connaissance de la capacité par unité de largeur [4]. $\beta$ représente le rapport entre le coefficient $\alpha$ et la partie imaginaire de l'impédance thermique. Compte tenu de ces valeurs numériques, il vient :

$$
\begin{gathered}
v_{\text {sat } \mathrm{MKSA}}=1,55 \cdot 10^{4} \cdot \exp \left(-1,9 \cdot 10^{-3}\left(T-T_{0}\right)\right), \\
200 \mathrm{~K}<T<400 \mathrm{~K} .
\end{gathered}
$$

Ainsi on retrouve dans le cas des trous sur le plan (111) un mode d'évolution exponentiel de la vitesse en fonction de la température. Rappelons que ce type de loi traduit les résultats expérimentaux plus anciens fournis par R. W. Coen et al. [3] dans le cas des électrons sur (100) et (111) et des trous sur (100), par F. F. Fang et al. [2] pour les électrons sur (100) et (111) et par M. Gamboa et al. pour les électrons sur (111) [4] (Fig. 3).

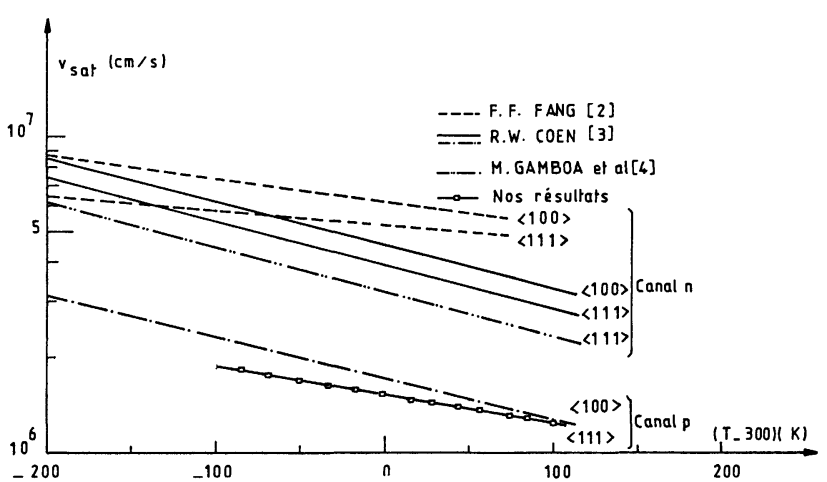

Fig. 3. - Résultats expérimentaux obtenus par d'autres auteurs et nous-mêmes.

[Experimental results obtained by other authors and ourselves.]

5. Conclusion. - Nous avons appliqué au cas des trous d'un canal inversé sur substrat $N$ (111) une méthode de détermination de la loi de variation de la vitesse limite en température [4]. La loi obtenue fait apparaître une dépendance exponentielle entre la vitesse limite et la température, ce qui semble être un mode d'évolution général indépendant de la nature des porteurs ou de l'orientation cristallographique. Les valeurs numériques proposées sont à notre connaissance les seules disponibles actuellement. 


\section{Bibliographie}

[1] Merckel, G., Une méthode simple de détermination de la vitesse limite des porteurs, dans un transistor MOS, Revue Phys. Appl. 15 (1980) 879-887.

[2] FANG, F. F. and Fowler, A. B., Hot Electron Effects and Saturation Velocities in Silicon Inversion Layers, J. Appl. Phys. 41, no 4 (1970) 1825-1831.

[3] Coen, R. W., Muller, R. S., Velocity of Surface Carriers in Inversion Layers of Silicon, Solid State Electron. 23 (1980) 35-40.

[4] Gamboa, M., Sarrabayrouse, G., Tranduc, H., Rossel, P., Influence de la température sur la vitesse limite des porteurs dans un transistor MOS à canal court, Revue Phys. Appl. 15 (1980) 973-975

[5] Rossel, P., Gamboa, M., Tranduc, H., Martinot, H., Influence de la contre-réaction thermique sur l'impédance de sortie des transistors MOS à canaux courts, Revue Phys. Appl. 14 (1979) 911-919.

[6] SigG, H. J., Vandelin, G. D., Cauge, T. P., Kocsis, J., D. MOS transistor for Microwave Applications, I.E.E.E. Trans. Electron Devices ED 19, no 1 (1972) 45-53. 